\title{
Essai de Géoblocs (B) pour la réparation et l'entretien des digues en enrochements
}

Olivier Briand

Docteur en sciences, Société Géomer, Marseille

Denis Gieulles

Directeur, Société Géomer, Marseille

Résumé :

Le Géobloc $\otimes$ est un concept simple qui consiste à couler du béton dans un coffrage souple (géoconteneur) en géotextile résistant afin de remplacer des blocs d'enrochements perdus ou de combler des trous apparus à la suite de mouvements de blocs. Cette technique de mise en oeuvre très rapide, s'applique avec peu de

personnel et des moyens matériels rudimentaires. Elle permet de combler des vides, sans avoir à déposer la carapace de l'ouvrage, ce qui réduit fortement les coûts d'entretien. Cette solution semble améliorer la stabilité générale de la carapace

\section{Introduction}

Depuis plusieurs années, nous avons procédé à des expertises annuelles de ce type d'ouvrage dans le cadre de contrats d'assurance. Souvent confrontés à la nécessité de préconiser des travaux de faible étendue, nous avons recherché des solutions simples et peu onéreuses. Les Géoblocs ß sont la principale solution que nous recommandons pour les petits travaux d'entretien ou de réparation.

L'exemple traité ici est celui de la digue du port des Engraviers à Bandol dans la copropriété Bandol-Athéna. La digue du port est récente puisqu'elle a été reconstruite en 1985 à la suite de sa ruine totale et que les premiers travaux d'entretients en géoblocs ont été réalisés en 1988 (Figure 1).

L'ouvrage a été dimensionné selon les règles de l'art et a fait l'objet d'essais de stabilité en canal à houle au L.C.H.F[1]. Les gestionnaires du port ont alors décidé de souscrire une assurance pour cette digue. Nous avons ensuite été mandatés pour réaliser les inspections annuelles suivant le contrat d'assurance spécifique proposé par les Mutuelles du Mans. Au cours de ces dix années écoulées des réparations ponctuelles d'entretien ont été réalisées, soit par apport de nouveaux blocs

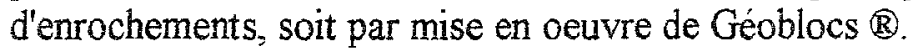




\section{Mise en oeuvre de Géoblocs @}

Le Géobloc $(B$ est un concept simple qui consiste à couler du béton dans un coffrage souple (géoconteneur) en géotextile résistant afin de remplacer des blocs d'enrochements perdus ou de combler des trous apparus à la suite de mouvements de blocs.

La nappe géotextile est préparée et cousue selon la forme souhaitée, puis elle est mise en place dans le trou et fixée sur les blocs environnants. Lorsque ce travail de préparation est terminé, la manche de la bétonnière est amenée dans le coffrage souple et l'on coule le béton prise mer (Photo $\mathrm{N}^{\circ} 1$ et $\mathrm{N}^{\circ} 2$ ).

La souplesse de la nappe coffrage permet au béton d'épouser la forme du vide, mais la faible élongation du géotextile permet de contrôler sa répartition (Photos $N^{\circ} 3$ et $N^{\circ} 4$ ).

Cette technique de mise en oeuvre très rapide, s'applique avec peu de personnel et des moyens matériels rudimentaires. Elle permet de combler des vides, sans avoir à déposer la carapace de l'ouvrage, ce qui réduit fortement les coûts d'entretien

\section{Conclusion}

Cette solution a permis de renforcer la stabilité de la carapace en favorisant le blocage des bloes entre eux sans modifier la perméabilité de la carapace, phénomène qui serait très préjudiciable à la stabilité. En effet la digue a subi une très grosse tempête le 6 janvier 1994 de probabilité au moins vingtennale qui a provoqué l'apparition de trous importants dans la carapace comme l'avait prédit le modele réduit du L.C.H.F.

Les zones qui avaient été entretenues par les Géoblocs $\&$ n'ont pas subi de détérioration. Cette solution simple d'entretien est donc fiable, facile de mise en oeuvre et peu onéreuse.

Des essais en laboratoire permettraient de quantifiier l'amélioration de la stabilité de la carapace et de définir la densité optimmale du nombres de géoblocs par unités de surface. On pourrait alors envisager d'utiliser cette technique dès la construction des ouvrages, en employant des bétons mélangés à des polymères expansifs.

Réference bibliographique :

[1] MANOUJIAN S. et ESCURIER C. - Etude de stabilité en canal - Digue de Bandol port Athéna - L.C.H.F, Août 1994 - p. 21 


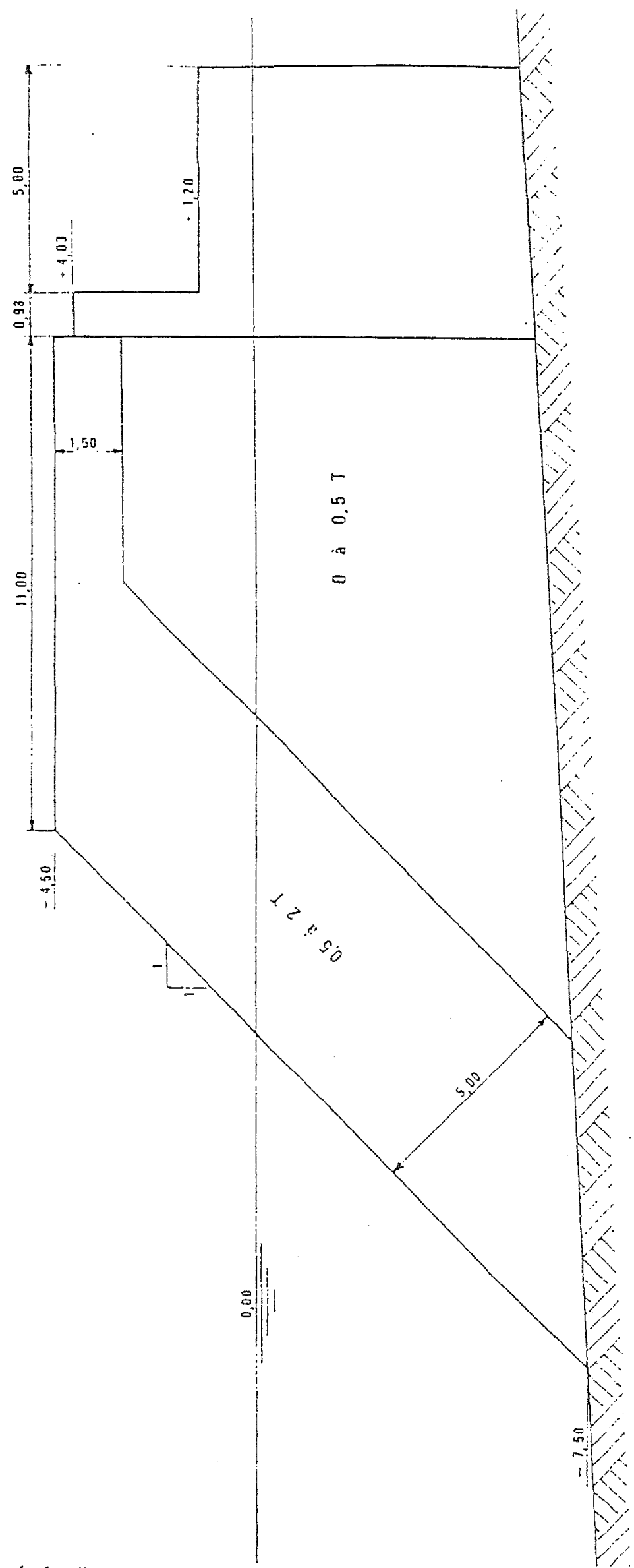

Figure 1 : Coupe type de la digue initiale - avant sa ruine en 1984 (Source [1]) 


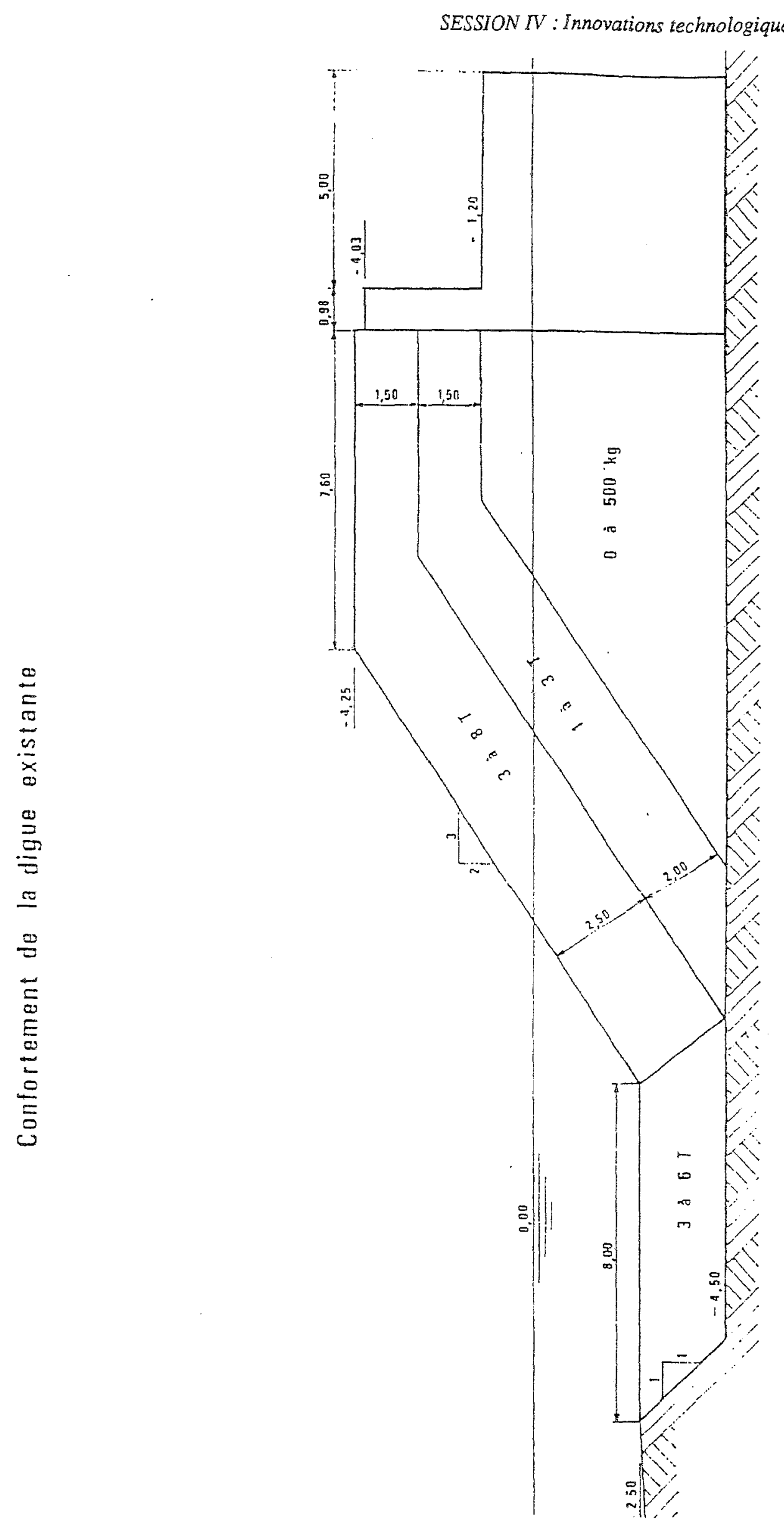

Figure 2 : Coupe type de la digue existante. (Source [1]) 


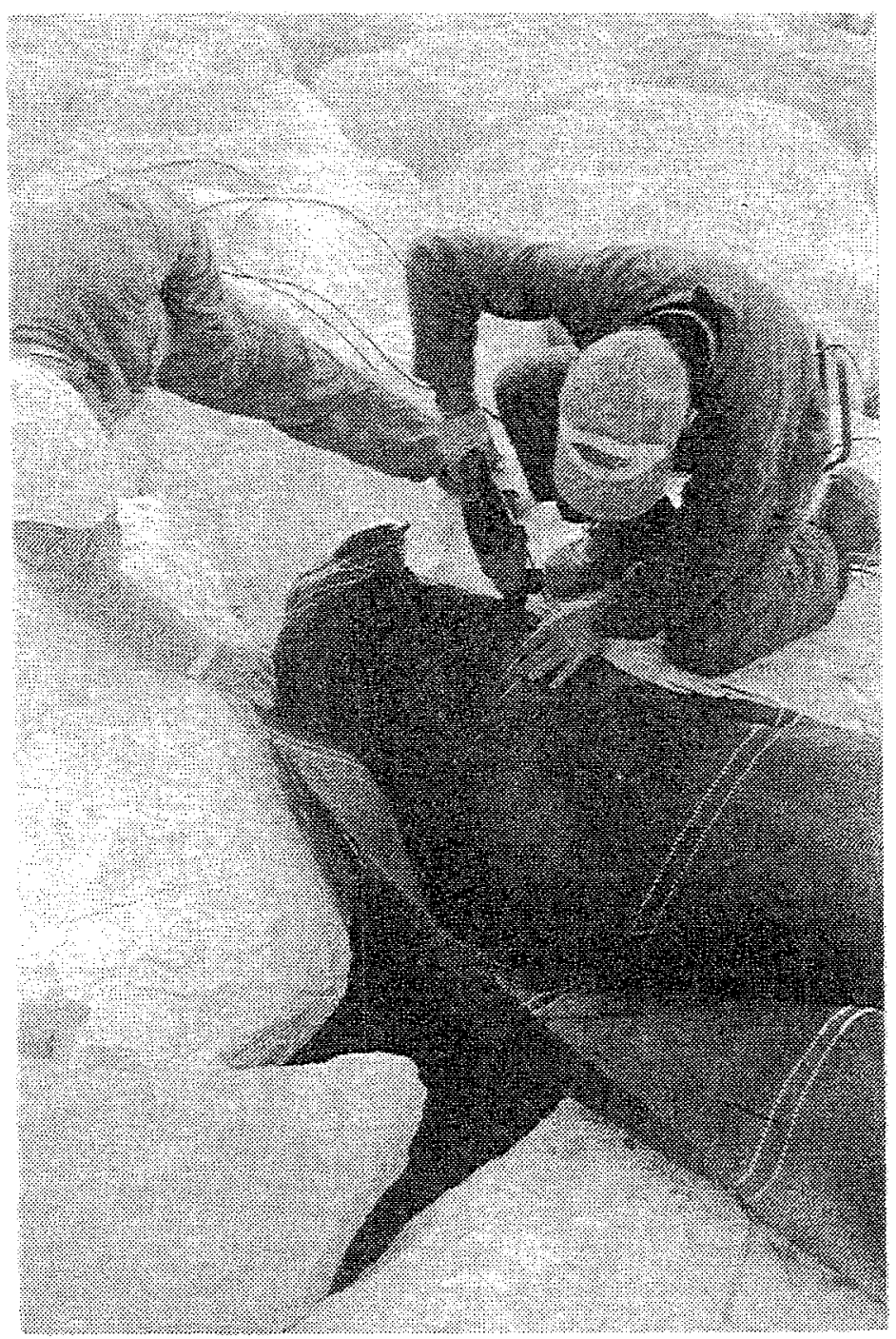

Photo $\mathrm{n}^{\circ} 1$ : Le sac de géotextile est spité sur les blocs environnants. 


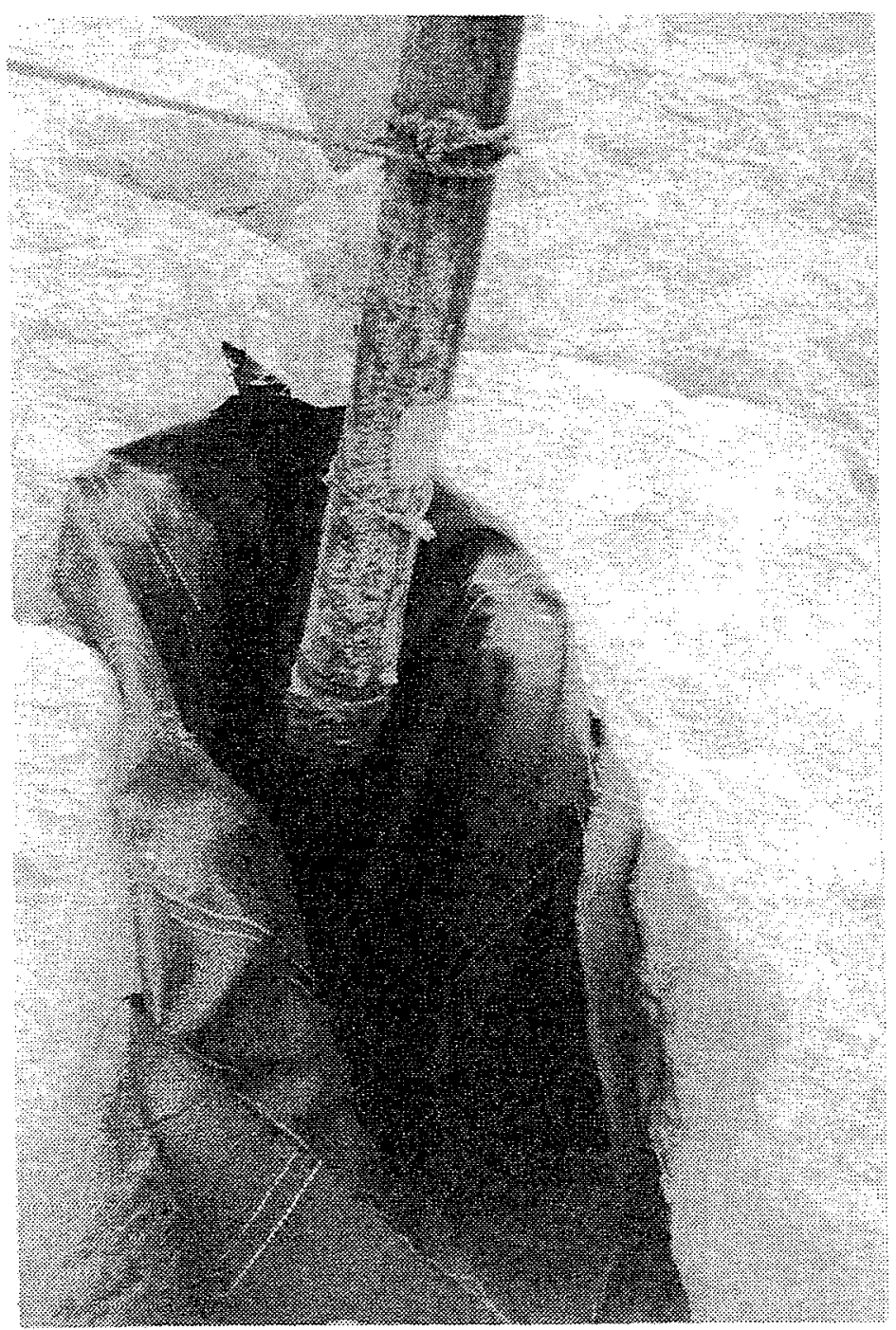

Photo $\mathrm{n}^{\circ} 2$ : Le sac en géotextile a été spité et le coulage du béton est en cours. 


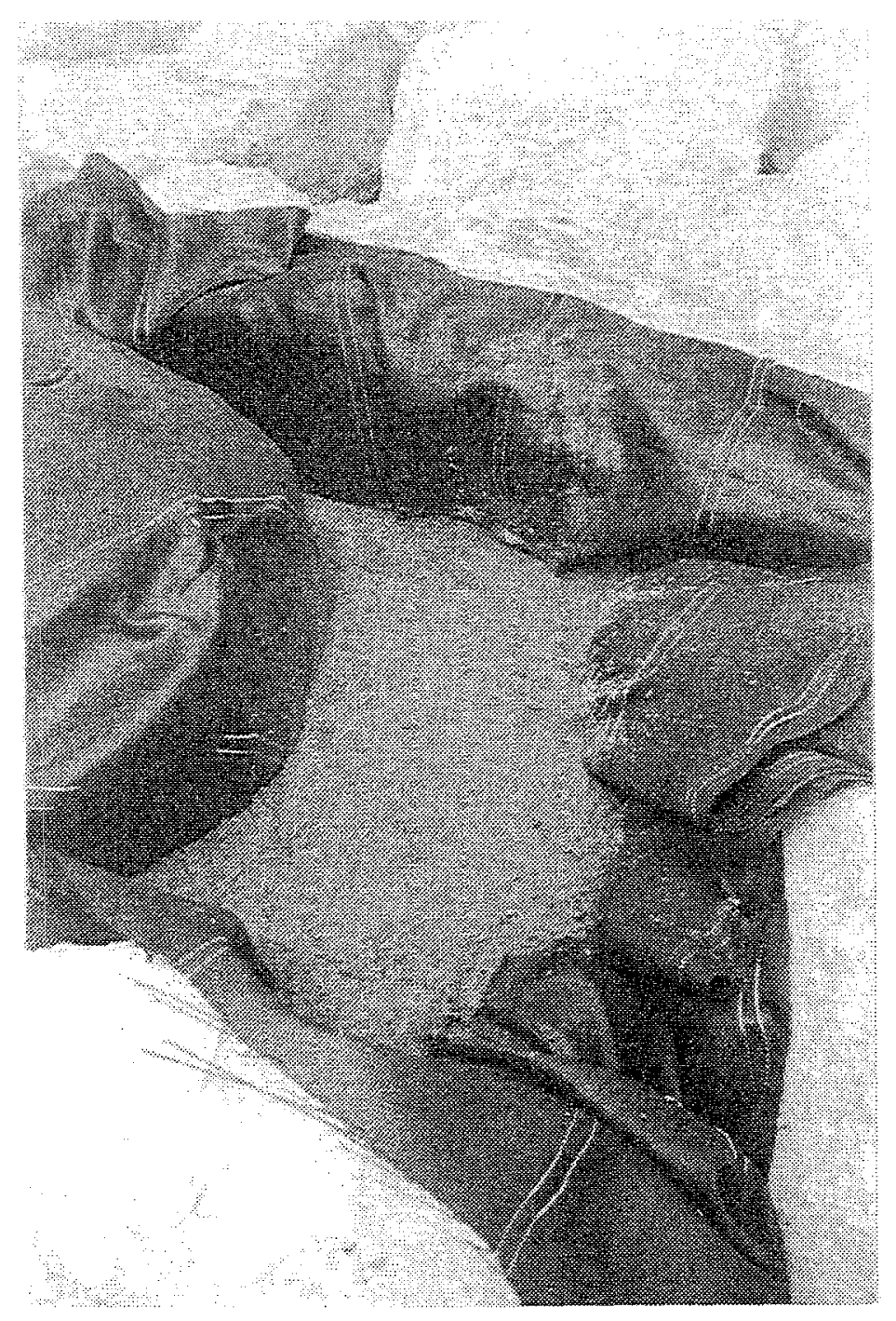

Photo $n^{\circ} 3$ : Le coulage est terminé ; le sac rempli de béton sera refermé afin de se prémunir contre le lessivage par l'eau de mer. 


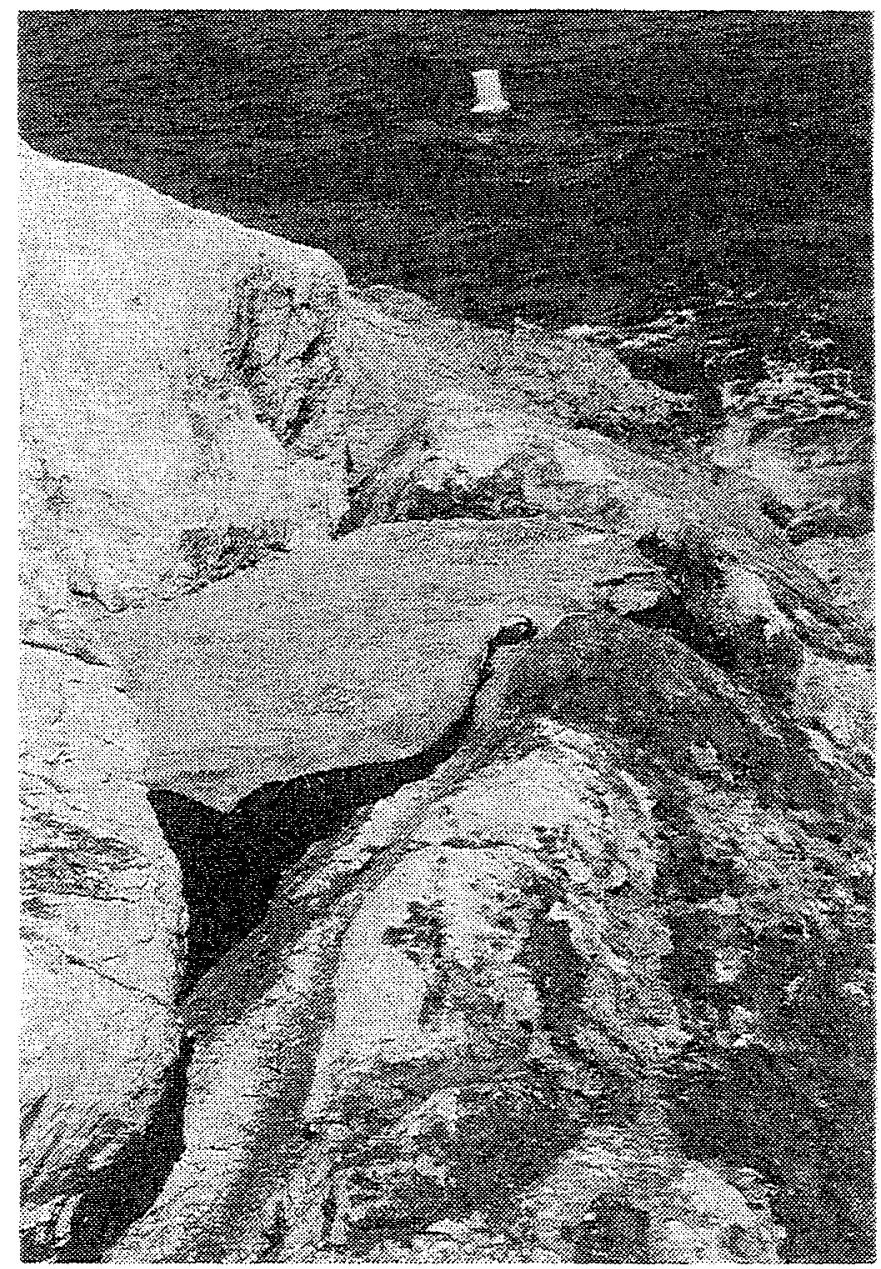

Photo $\mathrm{n}^{\circ} 4:$ Le Géobloc remplit les vides importants et il permet de pérenniser la stabilité de la carapace. 